\title{
Health-Related Quality of Life in Lung Transplantation Patients from Israel as Compared with General Population Data: A Pilot Study
}

\author{
Nissan Graur ${ }^{1,2 *}$ and Mordechai Reuven Kramer ${ }^{1,3}$ \\ ${ }^{1}$ Pulmonary Institute, Rabin Medical Center, Beilinson Hospital, Petach Tikva, Israel \\ ${ }^{2}$ Department of Physical Therapy, Faculty of Social Welfare and Health sciences, University of Haifa, Haifa, Israel \\ ${ }^{3}$ Sackler Faculty of Medicine, Tel Aviv University, Tel Aviv, Israel
}

\begin{abstract}
Lung transplantation (LT), one of the aims is improving patient's quality of life and survival. Our aim was to assess the impact of LT on health-related quality of life (HRQL) according to the SF-36 questionnaire in a group of 19 post-LT patients from Israel who had undergone lung transplantation more than one year prior to the study. The resulting data was compared with available norm data SF-36 questionnaire results reported in the general population. The results show that patients which were more than one-year (mean $13 \pm 5.1$ months) post-LT. As compared with norm data, post-LT patients reported significantly lower HRQL scores in 3 of the 8 health domains in the SF-36: Social functioning, physical health, and pain. The post-LT patients reported significantly higher HRQL scores in Vitality and Energy; higher mental health (emotional well-being) HRQL scores and similar HRQL scores in physical functioning, general and emotional health as compared with norm data. Compared with the general population, the significantly lower HRQL scores in 3 health domains as reported by our Israeli post-LT patients calls for additional multidisciplinary resources to support these patients' social functioning, physical health and improve the treatment of pain.
\end{abstract}

Keywords

Lung transplantation, Quality of life, SF-36 questionnaire

\section{Introduction}

Lung transplantation (LT) is an established treatment option for patients with a wide variety of end-stage lung diseases. Survival had been used as the main outcome measure after LT. However, currently one of the clinical aims of LT is to improve both survival and patients' health-related quality of life (HRQL) [1]. Nevertheless, exercise intolerance, functional disability, and peripheral muscle weakness often persist following LT [2-8]. Moreover, HRQL may be the only expected clinical benefit of LT in some indications as COPD, when extended survival is not assured [9]. HRQL is a patient-reported outcome that assesses the patient's perceptions of the impact of the disease and its treatment on their physical, social, functional, and emotional well-being. Numerous studies in recent years have reported on HRQL before and post-LT [10]. However, there is still paucity of studies on HRQL in post-LT patients, due to both the incidence of the procedure and the recipient's limited survival rates [11].

In Israel, this highly complex transplantation procedure is performed each year in 40-50 end-stage lung disease patients, only in one center, at the Rabin Medical Center, Beil- inson Hospital.

The objective of the present study was to assess the impact of lung transplantation on HRQL in a group of patients from Israel who had undergone lung transplantation more than one year, compared with available norm data from the general population.

\section{Patients and Methods}

Sixty Patients who had undergone lung transplantation

*Corresponding author: Dr. Nissan Graur, PT, PhD, Pulmonary Institute, Rabin Medical Center, Beilinson Hospital, Petach Tikva; Department of Physical Therapy, Faculty of Social Welfare and Health sciences, University of Haifa, Haifa, Hazait 84, Sitriya, 76834, Israel

Accepted: January 09, 2020

Published online: January 11, 2020

Citation: Graur N, Kramer MR (2020) Health-Related Quality of Life in Lung Transplantation Patients from Israel as Compared with General Population Data: A Pilot Study. J Transplant Surg 3(1):41-45 
Citation: Graur N, Kramer MR (2020) Health-Related Quality of Life in Lung Transplantation Patients from Israel as Compared with General Population Data: A Pilot Study. J Transplant Surg 3(1):41-45

Table 1: Demographic and clinical characteristics of the study group $(n=19)$.

\begin{tabular}{|l|l|}
\hline Age (years) & $\mathbf{5 2 \pm 1 3 . 9}$ \\
\hline Male/Female & $12 / 7$ \\
\hline Time from Transplantation (months) & $13 \pm 5.1$ \\
\hline Basic lung disease & \\
\hline Fibrosis & 5 \\
\hline Bronchiectasis & 2 \\
\hline Cystic Fibrosis & 5 \\
\hline Scleroderma & 1 \\
\hline Emphysema & 6 \\
\hline Type of Transplantation & \\
\hline SLT & 10 \\
\hline DLT & 9 \\
\hline BMI (index) & $23.8 \pm 5.7$ \\
\hline Weight (Kg) & $67.7 \pm 15.7$ \\
\hline Height (cm) & $168.9 \pm 10.9$ \\
\hline
\end{tabular}

Abbreviations: BMI: Body Mass Index; DLT: Double Lung Transplantation; SLT: Single Lung Transplantation. Data presented as means and standard deviations at the following measures: Age, Time from transplantation, BMI, Weight, and Height. All other measures are presented as frequencies.

more than one year prior to the study were assessed for eligibility, 41 were excluded (21 not meeting inclusion criteria and 20 declined to participate). They were recruited from the database of post lung transplant patients or at the outpatient clinic of the Pulmonary Institute, Rabin Medical Center, Beilinson Hospital, Petach Tikva, Israel.

\section{Inclusion criteria}

At least one year post-LT; clinically stable for at least one month.

Their clinical characteristics are summarized in Table 1.

\section{Study Design}

All the data were collected by the same investigator. The study protocol was approved by the institutional ethics committee (The study complied with the principles of the Declaration of Helsinki), and informed consent was obtained from all the subjects.

\section{Assessment of health-related quality of life}

The most popular generic HRQL instrument is the short form 36 item health status survey (SF-36). In the present study HRQL was measured by the Hebrew Short-Form (SF-36) Questionnaire [12] which has been used widely in many studies and health service institutions. The SF-36 features physical and mental summary scores, rating on a point scale from 0 to 100 points; the higher the score in each domain, indicates a better HRQL and a 4-point change in the SF-36 questionnaire domains is considered clinically significant.
The SF-36 questionnaire consists of 36- item questions, examining eight health domains:

1. Physical functioning: Limitations in physical activities because of health problems as limited mobility (walking down the street, up the stairs, shopping) and limited activities of daily living (bathing, and dressing etc. requiring assistance).

2. Physical health: Limitations in usual role/work activities because of physical health problems (e.g. lower job performance, necessity to terminate work because of exhaustion, etc.).

3. Emotional health limitations in usual role activities/work performance due to emotional health problems, such as depression and anxiety.

4. Vitality (energy/fatigue) - feelings of exhaustion, fatigue and pessimism, or experience high levels of energy and happiness.

5. General mental health (emotional well-being), situations where the mental and health condition limit social life and activity.

6. Social functioning: Limitations in social activities because of physical or emotional problems with family, friends and at work.

7. Bodily pain (pain)

8. General health perceptions (general health).

All study patients filled out the questionnaires independently. The methodological rules and data analysis from the SF-36 questionnaire have been described previously [13]. The resulting data on the HRQL of the post-lung transplant patients was compared to the published normative data in the general population based on results of the SF-36 questionnaire reported in the Medical Outcomes Study [14]. Notably, even in the general population the maximum result (100 points) is not achieved in any health domain.

\section{Data analysis}

Data was presented as means and standard deviations for the following measures: Age, time from transplantation, BMI, weight, and height. The obtained HRQL values were presented as means.

\section{Results}

Nineteen patients, 12 male and 7 females were recruited. The lung transplantation was performed in our study group of patients, mainly on patients with idiopathic pulmonary fibrosis (IPF) (5 patients, 26\%), emphysema (6 patients, $31 \%$ ) and cystic fibrosis (CF) (5 patients, $26 \%$ ). Our study group included 19 patients following LT aged 23-68 years-old, the youngest were the CF patients (Table 1).

A summary of the SF-36 questionnaire results and a comparison with the normal data from the general population are shown in Table 2.

In the physical functioning domain of the SF-36, the re- 
Citation: Graur N, Kramer MR (2020) Health-Related Quality of Life in Lung Transplantation Patients from Israel as Compared with General Population Data: A Pilot Study. J Transplant Surg 3(1):41-45

Table 2: Summary of the SF-36 questionnaire average scores in post-LT patients and in the general population.

\begin{tabular}{|l|l|l|l|}
\hline SF-36 Domains & $\begin{array}{l}\text { HRQL values in the study } \\
\text { group }\end{array}$ & $\begin{array}{l}\text { Average HRQL reported in } \\
\text { the general population }\end{array}$ & $\begin{array}{l}\text { The difference in the HRQL values between } \\
\text { post-LT patients and the general population }\end{array}$ \\
\hline Physical functioning & 69.21 & 70.61 & -1.4 \\
\hline Physical health & 48.68 & 52.97 & -4.29 \\
\hline Emotional health & 63.15 & 65.78 & -2.63 \\
\hline Vitality-energy/fatigue & 56.57 & 52.15 & 4.42 \\
\hline $\begin{array}{l}\text { Mental health/ } \\
\text { Emotional }\end{array}$ & 74.31 & 70.38 & 3.93 \\
\hline Well-being & & & \\
\hline Social functioning & 69.07 & 78.77 & -9.7 \\
\hline Pain & 67.76 & 70.77 & -3.01 \\
\hline General health & 57.10 & 56.99 & 0.11 \\
\hline
\end{tabular}

Categories with the highest difference in the quality of life between patients following LT and the general population are in bold.

sponders' average score of 69.21 can be classified as almost no difficulties with mobility and activities of daily living. Similar compared to the average score of 70.61 in the general population.

In the physical health domain of the SF-36, post-LT patients' average score of 48.68 , can be classified as mild limitations with work or activities, as a result of physical health problems. This was significantly lower compared to 52.97 , the average score in the general population.

In the emotional health domain of the SF-36, the responders' average score of 63.15 , can be classified as mild limitations with work performance due to emotional health problems. Compared to 65.78, the average score in the general population.

In the vitality - (energy/fatigue) domain of the SF-36, the post-LT patients' average score of 56.57, can be classified as no feelings of exhaustion and fatigue, rather experience slightly above average levels of vitality and energy. Which are significantly higher compared to the average score of 52.15 , in the general population.

In the general mental health (emotional well-being) domain of the SF-36, the responders' average score of 74.31, can be classified as no limitations in social activities due to mental health problems. Compared to 70.38 , the average score in the general population.

In the social functioning domain of the SF-36, the postLT patients' average score of 69.07, can be classified as mild to moderate reduction in social activities due to health and emotional problems. This was significantly lower compared to 78.77 the average score in the general population.

In the pain domain of the SF-36, the post-LT patients' average score of 67.76 , can be classified as a mild problem indicating the presence of pain. This was significantly lower compared to 70.77 , the average score in the general population.

In the general health domain of the SF-36, the responders' average score of 57.10, can be classified as no problem with general health. Similar to the average score of 56.99. in the general population.

\section{Discussion}

To the best of our knowledge, the present crossover study is the first to examine the impact of LT on HRQL in a group of patients from Israel, compared with available norm data from the general population. The participants were more than one-year (mean $13 \pm 5.1$ months) post-LT. The main findings show that adult post-LT patients have exhibited on average lower results in HRQL compared to the general population in HRQL. We found the most significant differences in HRQL compared to the general population were detected in 3 of the 8 health domains: Physical health (lower score by 4.29 points), social functioning (lower score by 9.7 points) and pain (lower score by 3.01 points, almost significant). While on average the study cohort stated significantly higher ratings compared to the general population in Vitality and Energy (higher score by 4.42 points), and similar ratings in physical functioning and emotional and general health. Together, these results could indicate positive improvement following LT.

The one-year benefits sustained regardless of sex, age, type of transplantation and basic lung disease. We observed an improvement post-LT in mental health (emotional well-being), higher than the general population by 3.93 points. It is important to note that SF-36 questionnaire measures global, not disease specific, HRQL. Our results show $L T$ does not confer significant HRQL benefits across all domains. Patients in our study reported higher mental health and vitality domain scores but reported no improvement in social functioning and physical health domains. This suggests that in our group of patients, undergoing lung transplantation had a greater impact on their vitality and mental health. More studies that extend beyond one year are needed to understand the impact of LT on HRQL in this population.

Improvement in HRQL is an important objective of the LT procedure. It has even been suggested that for diseases such as COPD, in which recipient's survival rates are lim- 
Citation: Graur N, Kramer MR (2020) Health-Related Quality of Life in Lung Transplantation Patients from Israel as Compared with General Population Data: A Pilot Study. J Transplant Surg 3(1):41-45

ited, improving HRQL is one of the primary goal of the LT procedure [9].

Long-term data has demonstrated improved HRQL after LT $[15,16]$. A two-year follow-up study of post-LT patients found significant improvements on seven of eight subscales in the SF-36, although similar to our results, scores remained below those of the general population [16]. Three to five years after $L T$, patients reported frequent affective and cognitive problems. Headaches and depression were also more common when compared with patients earlier in their transplant course [16]. These symptoms had a greater influence on women, resulting in a lower gain in quality of life compared to men [17].

Numerous studies have observed improved overall health and HRQL after LT and heart/lung transplantation (HLT) [18-22]. These gains in HRQL tend to be sustained for many recipients, but diminish with complications such as obliterative bronchiolitis (OB) $[19,21,23,24]$. Van Den Berg, et al. carried out extensive research to examine the relationship between diminished HRQL and the onset of $O B$ [21]. In their study, patients were assessed both cross-sectionally and longitudinally at four months post-transplantation. Although results demonstrated a true association between these events, a causal relationship was not proven [21].

The study by Anyanwu, et al. was one of the first to address the important issue of potential differences in $\mathrm{HRQL}$ according to the type of $L T$ procedure, comparing HRQL in single LT (SLT), double LT (DLT), and HLT recipients [22]. They conducted a cross-sectional study of 87 pre-transplant and 255 post-transplant patients and found a greater rate of HRQL improvement among the DLT and HLT recipients, relative to the SLT recipients. The baseline HRQL was found to be lower in the SLT recipients prior to transplant in comparison with the DLT and HLT patients [22]. It is possible that differences in the underlying disease or poorer general health prior to the transplant affected both the choice of transplant procedure and the different HRQL outcomes.

In summary, although the reports about HRQL in post-LT patients are favorable [25], more research is needed to better understand the specific causative factors affecting HRQL in LT recipients.

\section{Limitations of the Study}

1. The study results are limited due to the small number of participants combined with the high variability (heterogeneous) between participants, thus to corroborate the current findings, warrants conducting larger cohort studies. Langer, et al. [26] stated one limitation of their study was refusal of almost $40 \%$ of eligible candidates to participate. We agree, this low participation rate reflected in the small number of participants in these studies indicates the difficulty in motivating patients to attend participation in research studies following LT. Possible reasons are large travel distances to reach the transplant centers or the large spontaneous improve- ments, perceived by patients.

2. This study was not a longitudinal pre- and post-transplantation evaluation of patients' HRQL.

3. The side effects of anti-rejection medication and limitations this may bring to post-transplant patients' lifestyle brings an inherent difference to the HRQL of the general population who do not have these limitations or side effects. Further the effects of regular check-ups and limited life expectancy of lung transplant patients makes them difficult to compare to the general population.

\section{Conclusion}

Compared with the general population, the HRQL in our adult Israeli LT patients was lower in the following domains of the SF-36 questionnaire: Social functioning, physical health, and pain. Our results call for additional multidisciplinary resources to support patient social functioning, physical health and to make improvements in the understanding and in the treatment of pain. The results of our study could be used as a pilot study for a more extensive study of HRQL in post-LT population, numbers of participants could be raised and a more specific lung disease group targeted at several specific time points after the transplantation. Age differences and differences between SLT and DLT could be also detected.

\section{Financial Disclosure and Conflicts of Interest}

None of the authors have financial or other potential conflicts of interest to disclose.

\section{Acknowledgements}

The authors thank the study coordinators and nurses of the Pulmonary Institute for their help in the recruitment process, and the pulmonary function technicians for their professional assistance in the study. Finally, the authors extend thanks to Ms. Dalia Dawn Orkin for her important English language contributions.

\section{References}

1. Weill D, Benden C, Corris PA, et al. (2015) A consensus document for the selection of lung transplant candidates: 2014--an update from the Pulmonary Transplantation Council of the International Society for Heart and Lung Transplantation. J Heart Lung Transplant 34: 1-15.

2. Rochester CL (2008) Pulmonary rehabilitation for patients who undergo lung-volume-reduction surgery or lung transplantation. Respir Care 53: 1196-1202.

3. Reinsma GD, ten Hacken NH, Grevnik RG, et al. (2006) Limiting factors of exercise performance 1 year after lung transplantation. J Heart Lung Transplant 25: 1310-1316.

4. Van Der Woude BT, Kropmans TJ, Douma KW, et al. (2002) Peripheral muscle force and exercise capacity in lung transplant candidates. Int J Rehabil Res 25: 351-355.

5. Pantoja JG, Andrade FH, Stokic DS, et al. (1999) Respiratory and limb muscle function in lung allograft recipients. Am J Respir Crit Care Med 160: 1205-1211.

6. Pinet C, Scillia P, Cassart M, et al. (2004) Preferential reduction of 
quadriceps over respiratory muscle strength and bulk after lung transplantation for cystic fibrosis. Thorax 59: 783-789.

7. Williams TJ, Patterson GA, McClean PA, et al. (1992) Maximal exercise testing in single and double lung transplant recipients. Am Rev Respir Dis 145: 101-105.

8. Mathur S, Reid WD, Levy RD (2004) Exercise limitation in recipients of lung transplants. Phys Ther 84: 1178-1187.

9. Singer JP, Singer LG (2013) Quality of life in lung transplantation. Semin Respir Crit Care Med 34: 421-430.

10. Seiler A, Klaghofer R, Ture M, et al. (2016) A systematic review of health-related quality of life and psychological outcomes after lung transplantation. J Heart Lung Transplant 35: 195-202.

11. Kugler C, Gottlieb J, Warnecke G, et al. (2013) Health-related quality of life after solid organ transplantation: A prospective, multiorgan cohort study. Transplantation 96: 316-323.

12. Ware JE, Gandek B (1998) Overview of the SF-36 health survey and the International Quality of Life Assessment Project (IQOLA). J Clin Epidemiol 51: 903-912.

13. Jastrzębski D, Kozielski J, Banas A, et al. (2005) Quality of life during one-year observation of patients with idiopathic pulmonary fibrosis awaiting lung transplantation. J Physiol Pharmacol 56: 99-106.

14. Medical Outcomes Study. 36-Item short form survey (SF-36) scoring instructions.

15. Gerbase MW, Spiliopoulos A, Rochat T, et al. (2005) Health-related quality of life following single or bilateral lung transplantation: A 7-year comparison to functional outcome. Chest 128: 1371-1378.

16. Rodrigue JR, Baz MA, Kanasky JWF, et al. (2005) Does lung transplantation improve health-related quality of life? The University of Florida experience. J Heart Lung Transplant 24: 755-763.
17. Rodrigue JR, Baz MA (2006) Are there sex differences in health-related quality of life after lung transplantation for chronic obstructive pulmonary disease? J Heart Lung Transplant 25: 120-125.

18. Ramsey SD, Patrick DL, Lewis S, et al. (1995) Improvement in quality of life after lung transplantation: A preliminary study. The University of Washington Medical Center Lung Transplant Study Group. J Heart Lung Transplant 14: 870-877.

19. Gross C, Savik K, Bolman M, et al. (1995) Long-term health status and quality-of-life outcomes of lung transplant recipients. Chest 108: 1587-1593.

20. TenVergert EM, Essink-Bot ML, Geertsma A, et al. (1998) The effect of lung transplantation on health-related quality of life: a longitudinal study. Chest 113: 358-364.

21. Van Den Berg JW, Geertsma A, van Der BIJ, et al. (2000) Bronchiolitis obliterans syndrome after lung transplantation and health-related quality of life. Am J Respir Crit Care Med 161: 1937-1941.

22. Anyanwu AC, McGuire A, Rogers CA, et al. (2001) Assessment of quality of life in lung transplantation using a simple generic tool. Thorax 56: 218-222.

23. Gross CR, Raghu $G$ (1997) The cost of lung transplantation and the quality of life post-transplant. Clin Chest Med 18: 391-403.

24. Lanuza DM, Lefaiver C, Mc CM, et al. (2000) Prospective study of functional status and quality of life before and after lung transplantation. Chest 118: 115-122.

25. Ramsey S, Patrick D, Albert R, et al. (1995) The cost effectiveness of lung transplantation. Chest 108: 1594-1601.

26. Langer D, Burtina C, Schepersa L, et al. (2012) Exercise training after lung transplantation improves participation in daily activity: A randomized controlled trial. Am J Transplant 12: 1584-1592.

DOI: $10.36959 / 338 / 330$

Copyright: (c) 2020 Graur N, et al. This is an open-access article distributed under the terms of the Creative Commons Attribution License, which permits unrestricted use, distribution, and reproduction in any medium, provided the original author and source are credited. 